\title{
Mutilación genital femenina: un riesgo incrementado por la indiferencia social
}

\section{Female genital mutilation: an increased risk by social indifference}

\author{
Andrea Hernández-Quirama ${ }^{1}$; Hector-Mauricio Rojas-Betancur ${ }^{1}$
}

Forma de citar: Hernández-Quirama A, Rojas-Betancur HM. Mutilación genital femenina: un riesgo incrementado por la indiferencia social. Rev Univ Ind Santander Salud. 2019; 51(2): 169-173. doi: http://dx.doi.org/10.18273/revsal.v51n2-2019009 (c) (i)

\section{Resumen}

La Mutilación Genital Femenina (MGF) constituye una grave violación a los derechos humanos, de la mujer y de la niñez. Si bien se considera una práctica ancestral en algunas culturas, y es justificada y reproducida bajo el relativismo cultural, es un problema que requiere una mayor atención de las instituciones en general y de la salud pública en particular. Colombia es uno de los pocos países en el continente que reporta casos de prácticas de mutilación; sin embargo, el problema es mundial, no solo por los altos flujos de migración de población en alto riesgo en Europa y Norteamérica, sino porque es un imperativo global erradicar de una de las peores formas de violencia contra la mujer.

Palabras clave: Mutilación genital femenina; Salud pública; Niñez; Derechos humanos.

\begin{abstract}
Female genital mutilation constitutes a grave violation of human, women and children rights. It is considered an ancestral practice in some cultures, where it is justified and reproduced under the cultural relativism. It is a problem that requires greater attention of the institutions in general and public health in particular. Colombia is one of the few countries in America that reported cases of mutilation practices. However, the problem is global by large flows of high risk migrants to Europe and North America. Its eradication is a global imperative because it is one of the worst types of violence against women.
\end{abstract}

Keywords: Female genital mutilation; Public health; Children; Human rights.

\section{Introducción}

Dentro de las formas de violencia contra la mujer, la MGF es reconocida a nivel internacional como un tipo específico de violencia contra las niñas, las mujeres y contra los derechos humanos y de la mujer ${ }^{1}$. Se trata de prácticas de mutilación que afectan a niñas y mujeres, especialmente a las menores de 15 años, prácticas realizadas por razones que nada tienen que ver con una instrumentación médica o una justificación de bienestar

1. Universidad Industrial de Santander. Bucaramanga, Colombia

Correspondencia: Andrea Hernández. Calle 9 Carrera 27. Edificio de Administración 2 - Oficina 512. Teléfono: +57 7-6344000 ext 2847. Correo electrónico: ahernanq@uis.edu.co 
individual o colectivo, que se lleva a cabo en el marco de prácticas religiosas, culturales o costumbres ancestrales.

Por el contrario, la sociedad ha reaccionado sobre este tipo de violencias contra la mujer, aunque de manera reciente pues es sólo finalizando el siglo pasado, especialmente desde los años $70 \mathrm{~s}^{2}$, donde algunos movimientos sociales, comunidades académicas y organismos mundiales, inician la lucha contra este tipo de prácticas elevando su condición a una grave violación de los derechos más básicos ${ }^{3}$, enmarcando este tipo de prácticas como parte de las violencias contra las mujeres (VCM), y no simplemente como se denominaba antes simplemente como "circuncisión femenina".

La Organización Mundial de la Salud (OMS), define la MGF, como aquellas intervenciones sobre los genitales femeninos que producen la extirpación total o parcial o cualquier otra lesión de los genitales de la mujer, por razones sociales o culturales, sin ningún motivo médico ${ }^{4}$, estableciendo, de acuerdo a la gravedad de la mutilación, cuatro tipos:

\begin{tabular}{|c|c|c|}
\hline & Denominación & Definición \\
\hline Tipo I & Clitoridectomía & $\begin{array}{l}\text { Eliminación del prepucio del clítoris, con o sin escisión total o parcial del clítoris; este tipo se } \\
\text { conoce en el mundo islámico como sunna y equivale a lo que se denomina circuncisión, en tanto } \\
\text { que en África se equipara a la circuncisión masculina. }\end{array}$ \\
\hline Tipo II & Excisión & $\begin{array}{l}\text { Consiste en la extirpación del clítoris junto con la escisión total o parcial de labios menores, } \\
\text { dejando intactos los labios mayores. }\end{array}$ \\
\hline Tipo III & Infibulación & $\begin{array}{l}\text { Es la forma más severa de mutilación, con extirpación del clítoris y de los labios mayores y } \\
\text { menores, para después suturar ambos lados de la vulva dejando un pequeño orificio que permite la } \\
\text { salida de la orina y del flujo menstrual }\end{array}$ \\
\hline Tipo IV & Otros & $\begin{array}{l}\text { Todos los demás procedimientos lesivos de los genitales femeninos con fines no médicos, tales } \\
\text { como la punción, perforación, incisión, raspado o cauterización. }\end{array}$ \\
\hline
\end{tabular}

Fuentes $^{5,1}$

La definición de la OMS, llama la atención, además, sobre una práctica sin motivos médicos, realizada en pésimas condiciones de asepsia y de procedimientos que involucran personas no idóneas, instrumentos artesanales, como cuchillas y navajas, que incrementan los riesgos de muerte de las mujeres sometidas, riesgos hemorrágicos, riesgos de enfermedades de transmisión sexual, dolorosas y permanentes secuelas físicas para la mujer víctima de MGF.

Además, las diferentes definiciones ${ }^{6}$, coinciden en el daño psicosocial y en la prolongación de la mutilación durante el resto de la vida, sea como instrumentalización del control sobre la sexualidad femenina, sea en el valor social que esta práctica representa, los efectos de la MGF, como toda mutilación intencional, serán permanentes, estigmatizadores y recurrentes para las mujeres sometidas por razones como la disminución y el control del deseo sexual femenino, la fidelidad, el control sobre las relaciones maritales ${ }^{7}$, una supuesta estética genital o, incluso, una supuesta asepsia de la mujer que nació con unos genitales no congruentes con las ciertas creencias sociales.

De hecho, si bien la MGF se presenta especialmente en niñas menores de 15 años de edad, bajo el precepto de una corrección temprana de supuestos defectos o la prevención de ciertas conductas sexuales o morales no permitidas, los efectos serán constantes como el dolor crónico permanente durante la menstruación, hemorragias, infecciones, retención de orina, además de traumas psicosociales silenciados y acallados bajo el supuesto de consideraciones religiosas o culturales ${ }^{8}$.

Entre las consecuencias psicosociales prevalecen el síndromede estrés postraumático, trastornos deansiedad, pánico, supresión de la sensación y pensamiento $\mathrm{y}$, a veces intento de suicidio ${ }^{9}$, adicionalmente, las personas encargadas de estas prácticas son usualmente mujeres parteras o abuelas que, sin tener conocimiento médico, realizan la mutilación genética, cargando con el peso social y un señalamiento como victimarias por parte de la sociedad que condena el mantenimiento de una práctica que se considera no solo arcaica, salvaje, sino además, por fuera de cualquier consideración o tolerancia desde el enfoque de los derechos humanos, los derechos de la niñez y los derechos de la mujer ${ }^{10}$, aunque sea una tradición ancestral y merezca, desde el relativismo cultural, una consideración especial que requiere un tratamiento específico puesto que no hay verdades universales ${ }^{11}$. En todo caso, más allá de las consideraciones formales sobre la legitimidad de las prácticas culturales y el derecho de los pueblos al ejercicio soberano de sus hábitos, las mujeres no 
desean mutilar mujeres, tampoco conocen los riesgos ${ }^{12}$, pero están bajo el mandato de una comunidad que las obliga, según el precepto social que mantiene este tipo de tradiciones.

Una manera que se ha dispuesto para enfrentar este problema y de reducir los riesgos para las mujeres, es la medicalización de la Ablación/Mutilación Genital Femenina (A/MGF) bajo el supuesto de que un personal más idóneo (médicos, paramédicos, enfermeros y parteras certificadas) no sólo pueden tener un mejor conocimiento sobre la anatomía femenina y un manejo adecuado de la asepsia y del dolor, bajo el uso de anestesias, sino que pueden garantizar un bajo riesgo de salud para las mujeres. Sin embargo, la medicalización de la A/MGF, que se presenta como una alternativa a las insalubres prácticas culturales, especialmente en países africanos ${ }^{13}$, es tan cuestionable desde la propia ética médica, como lo son las prácticas médicas estéticas, sin motivos médicos como lo plantea la OMS, y tan extendidas en la medicina "occidental".

Pero, además, la medicalización de la A/MGF, especialmente se convierte en un dispositivo de "normalización" de la mutilación por el carácter institucional que imprime el personal de salud y porque existen incluso en algunos países africanos, clínicas certificadas para esta práctica ${ }^{13}$, agravando la pérdida de autonomía de la niña y de la mujer para ejercer su libertad de elección, su autonomía y el ejercicio de sus derechos básicos y obstaculizando la posibilidad de erradicación de la MGF como propósito universal.

Por otro lado, el problema de la MGF ha sido asociado básicamente a comunidades de países africanos, especialmente del África Subsahariana occidental, central y oriental, en países como Sudán, Djibouti, Somalia, Eritrea, Etiopía y, recientemente Indonesia se suma a la lista de países con mayor número de mujeres con MGF, al establecer un sistema de información sobre este tema. Sin embargo, dados los fuertes movimientos de migración actual de estas comunidades, el problema se traslada y se extiende prácticamente a todo el mundo, como sucede en Australia, Norteamérica y Europa ${ }^{7}$, puesto que cuando la gente emigra, lleva sus equipajes culturales y reproduce sus costumbres en el lugar en que se establece y las repuestas institucionales, por ejemplo en Europa, han sido poco significativas ${ }^{14}$.

Específicamente en el caso de la MGF y la migración, indica cómo el problema adquiere dimensiones globales y exige modelos de atención y de tratamiento unificados, que promueve la lucha contra la mutilación desde principios de universalidad, como su inclusión en las metas de los Objetivos de Desarrollo Sostenible (ODS) plantea a su vez, una serie de dilemas entre la aplicación de sistemas penales, el tratamiento humanitario y la condición de refugiados ${ }^{15}$.

Por otra parte, se vienen implementando diferentes acciones para que los sistemas de salud pública, especialmente en países con esta tradición y en países receptores de migración, como los europeos, tengan mejores herramientas para la prevención ${ }^{16}$, detección y tratamiento de la MGF; sumado a esfuerzos orientados a la educación para evidenciar y así aportar a mitigar los diferentes riesgos para las mujeres en esta situación ${ }^{17}$. Lo cual demuestra que se viene disponiendo de mejores prácticas del personal de salud para atender diferentes situaciones médicas y sociales en la atención del problema ${ }^{18}$.

Las estadísticas aún son muy incompletas respecto al tamaño del problema, pero se estima que al menos 200 millones de mujeres vivas, han sufrido MGF, en al menos 30 países que disponen hoy de estadísticas, sin embargo, el subregistro y el no registro impiden tener una información confiable sobre el problema, aunado al proceso de altas tasas de migración de poblaciones de estos países por diferentes razones ${ }^{6}$.

Precisamente organismos como el Fondo de Población de las Naciones Unidas (UNFPA), vienen promoviendo un importante trabajo para la eliminación de esta práctica, acudiendo en primer lugar a las propias comunidades involucradas y a los países que presentan mayor presencia de esta cuestionable tradición cultural, de hecho, se establecido la meta de la eliminación total de la MGF, en los objetivos del desarrollo sostenible para el año 2030.

Colombia es uno de los pocos países latinoamericanos que ha registrado casos de MGF, en comunidades indígenas ${ }^{19}$, aunque no hay datos precisos del número y localización de los mismos. Se visibilizó el problema en la comunidad indígena Emberá Chamí a raíz de la muerte de niñas víctimas de esta práctica en el año 2007, lo que provocó una respuesta institucional del Estado para sumarse a la lucha mundial para su erradicación e incluir el tema en la agenda nacional ${ }^{20-}$ ${ }^{22}$. En este sentido, se presentan distintos proyectos de intervención que intentan convencer a la propia comunidad de su responsabilidad, como comunidad autónoma, de acabar con esta práctica aprendida y trasmitida generacionalmente desde representaciones sobre la sexualidad y los derechos de las mujeres ${ }^{23}$. 
Posterior a la intervención interinstitucional realizada en la zona, las autoridades indígenas anunciaron la suspensión definitiva de la MGF. Sin embargo, algunos años después se volvieron a presentar registro de casos, lo que demuestra que eliminarla es un proceso que implica múltiples factores y por tanto puede tardar un tiempo considerable ${ }^{24}$. Pese a que exista un amplio referente normativo internacional y nacional que respalde su erradicación por violentar y vulnerar los derechos humanos y los derechos sexuales y reproductivos de las mujeres que son víctimas de la mutilación genital femenina ${ }^{25}$.

La MGF tiene variadas aristas de tipo cultural, social, salud, personal y familiar entre otros, que requieren ser tenidas en cuenta para su abordaje. En este sentido, es fundamental la revisión y reconfiguración desde diferentes ámbitos donde las mujeres tengan dominio y autonomía sobre su propio cuerpo y sexualidad.

\section{Discusión y conclusiones}

Existe un pleno consenso respecto al propósito de erradicar las violencias contra la mujer, donde la MGF, constituye una de las peores formas de violencia pues no solo se ejerce básicamente contra mujeres menores de 15 años de edad, las mujeres más indefensas, sino además que constituye una violencia extendida a toda su vida con graves consecuencias físicas y psicosociales.

Este consenso alcanza esferas y organismos mundiales, sin embargo, las medidas transnacionales y nacionales son bastante tímidas, las experiencias más significativas sobre la atención a la población víctima y en riesgo han sido básicamente locales ${ }^{16}$, donde se involucra el debate entre los universales deseables como los derechos humanos de la niñez y de la mujer y los objetivos de desarrollo sostenible, por una parte, y por otra, el relativismo cultural ${ }^{11}$, que pone de manifiesto el complejo entramado de situaciones alrededor de una problemática que, en principio, estaba altamente localizada en países considerados de bajo desarrollo o articulados con religiones o tradiciones lejos de los centros de poder.

No obstante, los altos flujos de migración, la medicalización de la MGF y los cambios en la cultura $^{13}$, ponen de manifiesto la importancia mundial del problema y su alta presencia, especialmente en los países europeos ${ }^{14}$, con un fuerte llamado de atención a los sistemas de salud para una preparación más humana del personal médico y paramédico en la atención de estas situaciones ${ }^{16}$.
Erradicar la MGF es un propósito que no solo alcanza consenso mundial, también significa que debe sumar esfuerzos no solo en el ámbito penal, sino especialmente en el cultural, en el ámbito de la prevención, la vigilancia epidemiológica y el involucramiento de las propias comunidades en donde la MGF ha sido una práctica ancestral.

En Colombia se ha evidenciado la práctica de MGF entre comunidades indígenas. No hay una explicación antropológica sobre el origen o trasmisión de esta práctica, tampoco existen registros claros sobre el número de casos o de comunidades que la practican. El Estado se ha comprometido en su erradicación y viene trabajando con la comunidad Emberá, donde se han presentado casos de muerte de niñas, desde un enfoque de autogestión comunitaria.

\section{Referencias}

1. Urdaneta E, Salcedo R. Mutilación genital en niñas: dolor, tristeza y sufrimiento en ellas. Rev Mex Pediatría. 2014; 81(1): 154-160.

2. Novak-Irons F. Mutilación genital femenina: un caso de asilo en Europa. Rev Migr Forzadas. 2015; 49: 77-79.

3. Simister J. Domestic violence and female genital mutilation in Kenya: effects of ethnicity and education. J Fam Viol. 2010; 25(3): 247-257. doi: https://doi.org/10.1007/s10896-009-9288-6.

4. WHO. Female genital mutilation. Fact sheet 241. Washigton: WHO; 2000.

5. WHO. Clasificación de la mutilación genital femenina.

6. United Nations Children's Fund. Female Genital Mutilation/Cutting: A global concern. New York: UNICEF, 2016.

7. Vizcaíno $\mathrm{M}^{\mathrm{a}}$. Mutilación genital femenina ¿práctica cultural o violencia de género? realidad en la comunidad Valenciana. Cult Cuidados. 2018; 22(50): 9-11. doi: http://dx.doi.org/10.14198/ cuid.2018.50.01.

8. Klein E, Helzner E, Shayowitz M, Kohlhoff S, Smith-Norowitz TA. Female genital mutilation: health consequences and complications. a short literature review. Obstet Gynecol Int. 2018; 1: 1-7. doi: https://doi.org/10.1155/2018/7365715.

9. Obaid Z, Amer A, El Mahdy M, Mohammed A. Evaluation of psychological and sexual effects of female genital mutilation (Circumcision). Egypt $\mathrm{J}$ Hosp Medicine. 2019; 4(1): 726-734. doi: 10.12816/ EJHM.2019.24073

10. Hermida del Llano C. La mutilación genital 
femenina desde la perspectiva jurídica española. Bajo Palabra. Rev Filosofía. 2017; 15: 47-66. doi: http://dx.doi.org/10.15366/bp2017.15.005

11. Prada A, Hermida del Llano C. La mutilación genital femenina. El declive de los mitos de legitimación. Valencia, Tirant lo Blanch 2017. Cuad Elect Filo Derecho. 2018; 38: 231-235. doi: https:// doi.org/10.7203/CEFD.38.1670.

12. Muchene K, Mageto I, Cheptum J. Knowledge and attitude on obstetric effects of female genital mutilation among Maasai women in maternity ward at Loitokitok Sub-County Hospital, Kenya. Obst Gynecol Int. 2018; 8418234: 1-5. doi: https://doi. org/10.1155/2018/8418234.

13. Foldes P, Martz F. La medicalización de la mutilación genital femenina. Rev Migrac Forzadas. 2015; 49: 82-83.

14. Christoffersen G, Bruhn P, de Neergaard R, Engel $\mathrm{S}$, Naeser V. Mapping the lack of public initiative against female genital mutilation in Denmark. Reprod Health. 2018; 15(1): 59-60. doi: https://doi. org/10.1186/s12978-018-0499-2.

15. Ropero Carrasco J. Inmigración, integración y diversidad: Un análisis crítico a partir del tratamiento de la mutilación genital femenina en la Unión Europea. Cuad Eur Deusto. 2017; 57(1): 133165. doi: https://doi.org/10.18543/ced-57-2017.

16. Waigwa S, Doos L, Bradbury-Jones C, Taylor J. Effectiveness of health education as an intervention designed to prevent female genital mutilation/cutting (FGM/C): a systematic review. Reprod Health. 2018; 15(1): 62-76. doi: https://doi.org/10.1186/ s12978-018-0503-x.

17. Alcón C, Jiménez I, Pastor M, Almansa P. Algoritmo de Actuación En La Prevención de La Mutilación Genital Femenina. Estudio de Casos Desde Atención Primaria. Atención Primaria. 2016; 48(3): 472-483. doi: https://doi.org/10.1016/j.aprim.2015.08.004.

18. Gallardo Y, Gallardo R, Núñez L. Mutilación genital femenina: elementos necesarios para su enfrentamiento. Revista Habanera de Ciencias Médicas. 2016; 5 (3): 472-483.

19. Unión Nacional de Asociaciones Familiares. Guía para profesionales. La MGF en España. Prevención e intervención.

20. Consejeria presidencial para los derechos humanos. Sumar esfuerzos y actuar: la clave para poner fin a la mutilación genital femenina en Colombia.

21. Consejeria presidencial para la equidad de la mujer. La mutilación genital femenina en Colombia, una práctica que vulnera los derechos humanos de las mujeres indígenas.

22. Instituto Colombiano de Bienestar Familiar. Acción interinstitucional para erradicar mutilación genital femenina.

23. Organización Nacional Indígena. Sumar esfuerzos y actuar: la clave para poner fin a la mutilación genital femenina en Colombia.

24. González Henao R. La ablación genital femenina en comunidades Embera chamí. Cuadernos Pagu. 2011; (37): 163-183. doi: http://dx.doi.org/10.1590/ S0104-83332011000200006.

25. Marrugo Nuñez N. Multiculturalismo, derechos humanos y derechos de los indígenas en Colombia. Práctica de la ablación en las niñas de la tribu Embera Chamí. Justicia Juris. 2014; 10(1): 32-42. 